Cita bibliográfica: García González, J. M., y Grande, R. (2018). Evaluación de la calidad de las estadísticas españolas de mortalidad a edades avanzadas (1910-2014). Boletín de la Asociación de Geógrafos Españoles, 76, 455-478. doi: 10.21138/bage.2530

\title{
Evaluación de la calidad \\ de las estadísticas españolas de mortalidad a edades avanzadas
}

Quality evaluation of Spanish mortality statistics at advanced ages

\author{
Juan Manuel García González
}

jmgargon@upo.es

Departamento de Sociología

Universidad Pablo de Olavide (España)

\author{
Rafael Grande \\ rgrande@uma.es \\ Departamento de Derecho del Estado y Sociología \\ Universidad de Málaga (España)
}

\section{Resumen}

El objetivo de este trabajo es evaluar la calidad de las estadísticas de mortalidad a edades superiores a 70 años en España desde 1910 hasta 2014. Se usan datos de mortalidad del INE y del Human Mortality Database (HMD) con los que se construyen dos tipos de indicadores de calidad: de exageración de edades y de redondeo de edades. Los resultados muestran que la calidad de las estadísticas de mortalidad a edades avanzadas mejora progresivamente en España a lo largo del siglo XX hasta que en la década de los ochenta se minimizan los errores, debido a la modernización y a la mejora en la recolección de datos del Registro civil.

Palabras clave: calidad de estadísticas; mortalidad; demografía; Registro civil; edades avanzadas. 


\begin{abstract}
The goal of this paper is to evaluate the quality of Spanish mortality statistics at advanced ages (70+) from 1910 to 2014. With data from INE and Human Mortality Database, we built two types of quality indicators: age heaping and age overstatement. Our results show that quality of Spanish mortality data at advanced ages improves throughout the 20th century. In the decade of the eighties, errors are minimized by modernization and by progress in data collection at the civil register.
\end{abstract}

Key words: quality of statistics; mortality; demography; civil register; old-age.

Agradecimientos: Este trabajo forma parte de dos proyectos I+D+i financiados por el Ministerio de Economía y Competitividad: «Asimilación laboral e integración social en flujos migratorios SurSur y Sur-Norte. El caso de América Latina y el Caribe» (Ref. CSO2014-57410-JIN) y «Familias, lugares y generaciones. Configuración espacial y generacional de los vínculos familiares de los mayores y consecuencias para la salud y el bienestar» (Ref. CSO2016-80908-R).

\title{
1 Introducción
}

La población de edades avanzadas se ha incrementado rápidamente en los países desarrollados desde principios del siglo XX. España no ha sido ajena a este fenómeno: la población mayor de 80 años se ha multiplicado por 15 y la población de centenarios lo ha hecho por 28. Incluso se ha producido la aparición de personas que han cumplido 110 o más años, denominadas supercentenarias.

Este cambio se ha debido a dos procesos entrelazados: la disminución de la mortalidad a todas las edades, pero especialmente a partir de los 65 años (Blanes, 2007); y el incremento de la esperanza de vida, Ilegándose a doblar al nacimiento desde inicios del siglo XX hasta la actualidad (García González, 2014). Durante los dos primeros tercios de siglo el principal contribuyente de esta transformación fue la disminución de la mortalidad infantil, que pasó de un 200 por mil en 1900 a un 18,9 por 1000 en 1975 (Gómez Redondo, 1992); y que alcanza un 2,8 por 1000 en 2015 (Instituto Nacional de Estadística, INE, 2015). En cambio, durante el último tercio del siglo XX fue el descenso de la mortalidad de 65 a 80 años, y en lo que llevamos de siglo XXI, la reducción a partir de los 80 años. De esta manera, la esperanza de vida al nacimiento en España en 2015 se ha situado en 79,9 años para los varones y en 85,4 para las mujeres (INE, 2016), una de las más altas del mundo. Por lo tanto, las edades avanzadas se han erigido en protagonistas de la evolución reciente de la mortalidad de la población española. La relevancia de estos números nos hace 
preguntarnos por la fiabilidad de los datos con los que se han calculado desde el siglo XIX: las estadísticas de mortalidad.

\subsection{Estado de la cuestión}

Desde 1564, las inscripciones de nacimientos (bautizos), matrimonios y defunciones (enterramientos) en España se llevan a cabo en los registros parroquiales. A partir de 1600 se comienzan a contabilizar las defunciones y no es hasta finales del siglo XVIII cuando se anota la edad de defunción, variable fundamental para el estudio de la mortalidad.

Las autoridades eclesiásticas mantienen esta tarea hasta que en la constitución de 1869 se reconoce el derecho a la libertad religiosa, punto de partida para la secularización de la inscripción de los eventos demográficos. Así, el Registro civil se implanta en España con la Ley Provisional de Registro civil de 17 de junio de 1870, y se complementa con el Reglamento de 13 de diciembre de 1870, de ejecución de las leyes de matrimonio y de Registro civil. Por lo tanto, desde 1871 todos los ciudadanos adquirieron la obligación de inscribir el nacimiento de sus hijos e hijas en el Registro civil de su municipio, que se adjudica a la administración judicial y que presenta tres características esenciales: independencia, una plantilla más especializada y una estructura territorial ya existente.

El Registro civil español se construye en paralelo al estado-nación burgués y a la descentralización municipal en una situación de constante inestabilidad política, económica y social, lo que le confiere determinados problemas. Nicolau (2005) afirma que las dificultades iniciales eran reconocidas por las mismas autoridades civiles, que denuncian la falta de medios administrativos y de personal verdaderamente cualificado, y la resistencia de la Iglesia a la cesión de competencias de inscripción. Por lo tanto, su verdadera consolidación no se consiguió hasta bien entrado el siglo XX (Reher y Valero, 2005). Algunos trabajos concluyen, además, que la calidad de los registros parroquiales superaba a la del incipiente Registro civil a finales del siglo XIX (Pérez Moreda, 1980; Brel, 1999), si bien este era considerablemente más exhaustivo que los primeros, dada su universalidad y obligatoriedad.

La calidad de los datos de mortalidad a edades avanzadas se ha estudiado abundantemente en la literatura internacional, ${ }^{1}$ si bien la comparación con España se complica dada la diversidad histórica y de funcionamiento del registro de las estadísticas vitales. No obstante, los trabajos de Väinö Kannisto y colegas (Kannisto, 1999; Thatcher, Kannisto y Vaupel, 1999) sentaron las bases

1 En Canadá, Bourbeau y Lebel (2000) y Bourbeau y Desjardins (2002, 2006); en China, Wang et al. (1999); en Estados Unidos, Anderson y Silver (1997) y Preston, Elo y Stewart (1999); en la URSS, Bennett y Garson (1983); en Suecia, Lundström (1995); en Dinamarca, Skytthe y Jeune (1995) y Skythe, Hauge y Jeune (1995), entre muchos otros. 
analíticas, de las que surgió el trabajo metodológico y de comparativa internacional de Jdanov et al. (2008).

La larga tradición de la demografía histórica en España ha suscitado un enorme interés por el estudio de la calidad del conjunto de las estadísticas vitales (Viciana, 1998; Reher y Valero, 2005; Nicolau, 2005; Cusidó, 2011), especialmente se ha estudiado la fecundidad y los nacimientos (Leasure, 1963; Livi-Bacci, 1968) y la mortalidad infantil (Pascua, 1934a; Arbelo, 1962; Gómez Redondo, 1984, 1992; Ramiro, 1998). Sin embargo, las evaluaciones de las estadísticas de mortalidad general (Pascua, 1934b; Pérez Moreda, 1980; Bernabeu, 1992; Blanes, 2007) y de mortalidad a edades avanzadas (Pastor, 1999; Jdanov et al., 2008; Jdanov y Jasilionis, 2010) escasean en mayor medida.

\subsection{Objetivo}

Dado este contexto, y tras comprobar la escasez de una literatura exhaustiva, se evidencian dos asuntos. Primero, que el debate sobre la calidad de los datos de mortalidad a las edades avanzadas se encuentra abierto. Segundo, que se necesita profundizar en el mismo para que los investigadores reconozcan las dificultades a las que se enfrentan a la hora de realizar este tipo de estudios y que de esta manera puedan proponer las soluciones adecuadas.

Este trabajo se marca el objetivo de evaluar la calidad de los datos de mortalidad a edades avanzadas en la población española desde 1910 a 2014 a nivel nacional y diferenciada por sexo, y analizar las causas que explican esa calidad. El concepto de edades avanzadas es difuso y no hay un estándar en el ámbito sociodemográfico. En esta investigación se definen como aquellas iguales o superiores a 70 años. Si bien la perspectiva más conservadora suele considerar a los 80 años como el inicio de esas edades, es necesario tener en cuenta la amplitud del período estudiado y la modificación del concepto de edad avanzada a lo largo del mismo. No olvidemos que a principios de siglo XX la esperanza de vida al nacimiento apenas superaba los 40 años y que no se alcanzaron los 70 años hasta mediados de los años sesenta.

\section{Métodos y datos}

\subsection{Datos}

En aras de posibilitar la comparación con otros países o territorios, hemos acudido a la base de datos Human Mortality Database (HMD). El HMD se elabora desde el Max Planck Institute for Demographic Research (Alemania) y la University of California, Berkeley (Estados Unidos), y proporciona datos y tablas de mortalidad estandarizadas a partir de un protocolo común (Wilmoth et al., 2007). La fiabilidad de las tablas se sustenta en dos puntos: por un lado, se construyen a partir de las tablas de mortalidad oficiales proporcionadas por los institutos oficiales de cada país (el 
INE en el caso de España); por otro lado, se someten a un proceso de reconstrucción (por el método del ratio de supervivencia, de Thatcher, Kannisto y Andreev (2002)) y de suavizado a partir del último intervalo de edad, variable en el tiempo y que generalmente ha tenido un valor inicial de 90 ○ 100 años de edad.

En primer lugar, se usan tablas de mortalidad por sexo desde 1910 a 2014. Hemos tomado tablas 1x1 (edades simples, año simple) y 1x10 (edades simples, decenales) para el período 1910-2014, y 1x5 (edades simples, quinquenal) para el período 2010-2014. Todas las tablas tienen un intervalo final abierto de 110 y más años de edad.

En segundo lugar, se usan datos de defunciones y de población expuesta al riesgo de morir para el mismo período. Frente a los recuentos clásicos de población (censo, padrón o las más recientes estimaciones de la población actual, en el caso de España), la población expuesta al riesgo de morir se basa en esos datos clásicos, pero incluye una pequeña corrección de continuidad para reflejar el ritmo de fallecimientos en cada período temporal. ${ }^{2}$

\subsection{Indicadores de calidad de los datos de mortalidad a edades avanzadas}

Siguiendo el trabajo de Thatcher, Kannisto y Vaupel (1998), se entiende por datos de buena calidad a edades avanzadas aquellos que cumplen los siguientes siete requisitos: un registro civil (o similar) consolidado; registro obligatorio del nacimiento; sin evidentes exageraciones de edad de defunción; sin indicios de redondeo en la edad de defunción; con ratios por sexo verosímiles; con consistencia interna de las tasas de mortalidad, del patrón por edad y del ratio por sexo a lo largo del tiempo; y que permitan comparar con otros países o con tendencias internacionales.

Los indicadores demográficos que se utilizan en la evaluación de la calidad de los datos de mortalidad suelen interpretarse mediante la comparación con una población de referencia, comúnmente denominada como golden standard, que represente los datos de mejor calidad. Jdanov et al. (2008) consideran que la población de Suecia es la más adecuada para actuar como población estándar de acuerdo a tres criterios. Primero, los datos demográficos de Suecia presentan una calidad contrastada desde 1750 y se consideran los de mejor calidad del mundo (Wang et al., 1999). Segundo, hay una gran tradición de registros civiles y parroquiales: el primer registro de población a nivel nacional se estableció en Suecia en 1631. Y tercero, la población sueca ya ha completado la última fase de la transición de la mortalidad. No obstante, a pesar de la calidad de los datos suecos, debemos ser conscientes de que están sujetos a modificar su estatus de golden standard en largas evoluciones temporales como la que se presenta en este manuscrito. Por lo tanto, es necesario analizar las relaciones de calidad de datos entre poblaciones con cautela,

2 Véase Wilmoth et al. (2007) para mayor información sobre el procedimiento. 
especialmente cuanto menores sean las diferencias. Dicho esto, en este estudio comparamos los indicadores de España con los de Suecia, asumiendo que los datos son de buena calidad cuando los primeros sigan el patrón de ese golden standard.

En concreto, hemos desarrollado una evaluación de la calidad de los datos de mortalidad a edades avanzadas en España mediante los primeros cuatro criterios de Thatcher, Kannisto y Vaupel (1998) que indicamos previamente. En los casos de la necesidad de un registro civil consolidado y de un registro obligatorio de nacimiento hemos acudido a la literatura y fuentes documentales existentes. Para descubrir patrones irregulares en las edades de defunción (exageración y redondeo) a partir de los 70 años utilizamos unos indicadores demográficos que es preciso definir a continuación. Jdanov et al. (2008) apuntan que cada una de esas medidas se refieren a un aspecto específico de los problemas de calidad de datos y que, además, no son las únicas, de modo que se necesitaría una aproximación más amplia para obtener conclusiones finales sobre la fiabilidad de los datos de mortalidad.

\section{Indicadores de exageración de edades de defunción}

La exageración de edades se produce cuando se declara una edad de fallecimiento mayor a la que realmente ha ocurrido. El problema de la exageración de edades de defunción conlleva el riesgo de obtener estructuras por edad ilógicas para el contexto sociodemográfico y de mortalidad existente (Kannisto, 1999), peligro que se incrementa cuanto más alta es la edad de fallecimiento. Examinamos dos indicadores para medir la existencia de exageración de edades.

El primer indicador parte del ratio del total de años-persona vividos a los 100 y a los 80 años de edad (T100/T80), que se divide por el mismo ratio del golden standard para obtener el índice final. Seguimos los criterios de Jdanov et al. (2008) para medir la calidad de los datos: $(0-1,49)$, buena calidad; $(1,50-1,99)$, calidad aceptable; $(2,00-3,99)$, calidad aceptable, pero con reservas; $(\geq 4,00)$, baja calidad.

Cabe apuntar que puede darse el caso de que no haya exageración de edades, sino que la mortalidad a los 80 y a los 100 años del territorio objeto de estudio sea menor que la del golden standard (Cheung y Robine, 2007). Esta situación suelen ocurrir en países que han experimentado una reducción más acelerada de la mortalidad a edades avanzadas que en el golden standard o en los que las tasas de mortalidad a esas edades son inesperadamente bajas a pesar de haber tenido una alta mortalidad en las edades jóvenes y adultas (Coale y Kisker, 1986). Para solventar este posible problema, Jdanov et al. (2008) proponen un criterio gráfico adicional que relacione la esperanza de vida a los 80 años con la probabilidad de supervivencia de 10 a 55 años. Uniendo ambos indicadores, hay exageración de edades si el ratio anterior es mayor que 1,5 y si los valores 
de la esperanza de vida a los 80 años son altos, a pesar de haber tenido una alta mortalidad jovenadulta (Jdanov et al., 2008).

El segundo indicador es el ratio de defunciones entre dos grupos de edad a la defunción: 105+ frente a 100+ (D105+/D100+), y 110+ frente a 105+ (D110+/D105+) (Kannisto, 1994, 1999), que se comparan con los correspondientes ratios del golden standard. Seguimos los criterios de Jdanov et al. (2008) para medir la calidad de los datos. Por un lado, para el ratio D105+/D100+: $(0,0-5,9)$, buena calidad; $(6,0-9,9)$, calidad aceptable; $(10,0-14,9)$, calidad aceptable pero con reservas; $(\geq 15,0)$, baja calidad. Por otro lado, para el ratio D110+/D105+: (0-9,9), buena calidad; $(10,0-14,9)$, calidad aceptable; $(15,0-24,9)$, calidad aceptable pero con reservas; $\geq$ 25,0), baja calidad.

2. Indicadores de redondeo y preferencia de dígitos en las edades de defunción

El redondeo se produce cuando hay una predilección por dígitos concretos (en especial, 0 y 5) a la hora de registrarse la edad de defunción. Examinamos dos indicadores para medir la existencia de redondeo.

Primero, el índice de Whipple mide la precisión de los datos en torno a una edad de defunción determinada acabada en un dígito susceptible de redondeo. Se calcula como el ratio de la suma de las defunciones cuyas edades acaban en 0 o 5 entre el total de defunciones dentro de un intervalo de edad concreto. En este trabajo calculamos la versión de este indicador que proporcionan Wang et al. (1999) para centenarios (100 o más años), y la adaptamos para nonagenarios (de 90 a 99 años).

Índice de Whipple $=\frac{\left(D_{x-5}+D_{x}+D_{x+5}\right) * 100 * 5}{\sum_{i=x-7}^{x+7} D i}$, donde $\mathrm{x}=$ edad de defunción, $\mathrm{D}=$ número de defunciones.

Para medir la fiabilidad de los datos seguimos dos criterios. Por un lado, el de Spoorenberg (2007) se aplica al valor del índice: ( $\leq 105)$, datos muy fiables; (105-110), datos fiables pero con reservas; (111-125), datos aceptables; (126-175), datos poco fiables; $\geq 176)$, datos no fiables. Por otro, la diferencia relativa entre el índice de Whipple del país que analizamos y el del golden standard (Jdanov et al., 2008). Para mujeres: (0,0-4,9), buena calidad; (5,0-8.9), calidad aceptable; (9,011,9), calidad relativamente aceptable; $(\geq 12,0)$, baja calidad. Para varones cambia ligeramente: (0,0-4,9), buena calidad; (5,0-9.9), calidad aceptable; (10,0-14,9), calidad relativamente aceptable; $(\geq 15,0)$, baja calidad. 
Segundo, usamos el índice de redondeo de edades que Kannisto (1999) propone para cualquier edad y que Wang et al. (1999) modifican para las edades 90 y 100, y que hemos adaptado para cualquier edad de defunción. Lo calculamos para 70, 75, 80, 85, 90, 95 y 100 años.

$$
\text { Indice de redondeo }=\frac{D_{i}}{\exp \left(\frac{1}{5} \sum_{x=i-2}^{i+2} \ln \left(D_{x}\right)\right)} \text {, donde } \mathrm{x}=\text { edad de defunción, } \mathrm{D}=\text { número de defunciones. }
$$

Para medir la existencia de redondeo seguimos el criterio de Kannisto (1999): $(<1,1)$, no hay redondeo; (1,1-1,2), niveles moderados de redondeo; $>$ 1,2), problemas de redondeo.

\section{Resultados}

Antes de comenzar a exponer los resultados principales de la evaluación de la calidad de los datos de mortalidad a edades avanzadas en España, conviene recordar que el Registro civil en España comienza a funcionar oficialmente en 1871. De este modo podemos establecer equivalencias entre el nacimiento y la defunción para todas las generaciones que fallecieron entre 1910 y 2014 con una edad entre 70 y 110 años o más. Diferenciamos dos tipos de generaciones. Primero, aquellas nacidas antes de 1871, que fallecieron entre 1939 y 1979. Segundo, aquellas nacidas en 1871 o más tarde, que fallecieron entre 1940 y 2014. Por ejemplo, una persona nacida en 1900 y que falleciera con 93 años, se habría inscrito en el Registro civil, pero no así una que hubiera nacido en 1867, fallecida a la misma edad en 1960. Esta relación entre fechas se torna fundamental a la hora de analizar el resto de indicadores de calidad de los datos de mortalidad.

\subsection{Exageración de la edad de defunción}

El primer indicador para evaluar la existencia de exageración de edades a la defunción es el ratio del total de años-persona vividos a los 100 y a los 80 años de edad (T100/T80), dividido por el mismo ratio en el golden standard.

En el caso de las mujeres, hay exageración de edades a la defunción en la primera mitad del siglo XX, con índices que superan ampliamente el límite de 4. Esta baja calidad continúa en la década de los años cincuenta y algo menos en la de los sesenta. Sin embargo, a partir de los años 70 la calidad de los datos se equipara a la de Suecia, con un ligero repunte del índice con la llegada del siglo XXI y, sobre todo, en el lustro 2010-2014, cuando supera de nuevo el valor 1,5 (Tabla 1). Por su parte, en los varones se repite un patrón relativamente similar: hay baja calidad hasta los años sesenta y una calidad aceptable desde entonces. Nótese que sólo en la década de los años setenta se consigue alcanzar una calidad similar a la sueca (Tabla 1). Desde los años setenta, y en comparación con las mujeres, hay un mayor nivel de exageración de edades. Esto se debe probablemente a las bajas cifras poblacionales de varones centenarios tanto en Suecia como en 
España (Kannisto-Thatcher Database on Old-Age Mortality, 2016), lo que podría tergiversar ambos ratios y, por consiguiente, el índice final.

Tabla 1. Ratio de años-persona vividos a los 100 y a los 80 años de edad.

España y Suecia. 1910-2014. Mujeres y varones

\begin{tabular}{|l|c|c|c|c|c|c|}
\hline \multirow{2}{*}{ Año } & \multicolumn{4}{|c|}{ Mujeres } & \multicolumn{3}{c|}{ Varones } \\
\cline { 2 - 7 } & España & Suecia & España/Suecia & España & Suecia & España/Suecia \\
\hline $1910-1919$ & 0,0061 & 0,0007 & 8,65 & 0,0019 & 0,0004 & 5,25 \\
\hline $1920-1929$ & 0,0066 & 0,0008 & 8,57 & 0,0020 & 0,0004 & 4,67 \\
\hline $1930-1939$ & 0,0054 & 0,0006 & 8,99 & 0,0013 & 0,0004 & 3,04 \\
\hline $1940-1949$ & 0,0039 & 0,0008 & 4,80 & 0,0011 & 0,0005 & 2,21 \\
\hline $1950-1959$ & 0,0034 & 0,0010 & 3,38 & 0,0013 & 0,0006 & 2,25 \\
\hline $1960-1969$ & 0,0030 & 0,0016 & 1,93 & 0,0013 & 0,0008 & 1,57 \\
\hline $1970-1979$ & 0,0027 & 0,0031 & 0,85 & 0,0015 & 0,0014 & 1,01 \\
\hline $1980-1989$ & 0,0041 & 0,0039 & 1,05 & 0,0025 & 0,0016 & 1,54 \\
\hline $1990-1999$ & 0,0049 & 0,0045 & 1,09 & 0,0028 & 0,0018 & 1,61 \\
\hline $2000-2009$ & 0,0067 & 0,0051 & 1,31 & 0,0036 & 0,0021 & 1,74 \\
\hline $2010-2014$ & 0,0095 & 0,0061 & 1,57 & 0,0053 & 0,0026 & 1,99 \\
\hline
\end{tabular}

Leyenda: (0-1,49), buena calidad; $(1,50-1,99)$, calidad aceptable; $(2,00-3,99)$, calidad aceptable, pero con reservas; $(\geq 4,00)$,

Fuente: elaboración propia a partir de datos de HMD

En cualquier caso, cabe preguntarse si hay realmente una sobreestimación de edades de defunción entre los españoles con respecto a los suecos. Para ello, comprobamos la relación entre la esperanza de vida a los 80 años y la supervivencia de los 10 a los 55 años. En la Figura 1, correspondiente a las mujeres, las españolas superan a las suecas desde inicios del siglo XXI, lo que explicaría la supuesta peor calidad de los datos con respecto al golden standard. En la Figura 2, correspondiente a los varones, podemos ver que el aumento de la esperanza de vida a los 80 años ha sido más acelerado en España que en Suecia a partir de los años setenta, lo que explicaría las divergencias en el indicador anterior. Por lo tanto, podemos afirmar que desde la década de los años setenta realmente no hay exageración de edades a la defunción en España, sino que se ha producido una mejora de la mortalidad de la población española con respecto a la sueca. 
Figura 1. Relación entre la esperanza de vida a los 80 años y la supervivencia de los 10 a los 55 años en España y Suecia. Mujeres

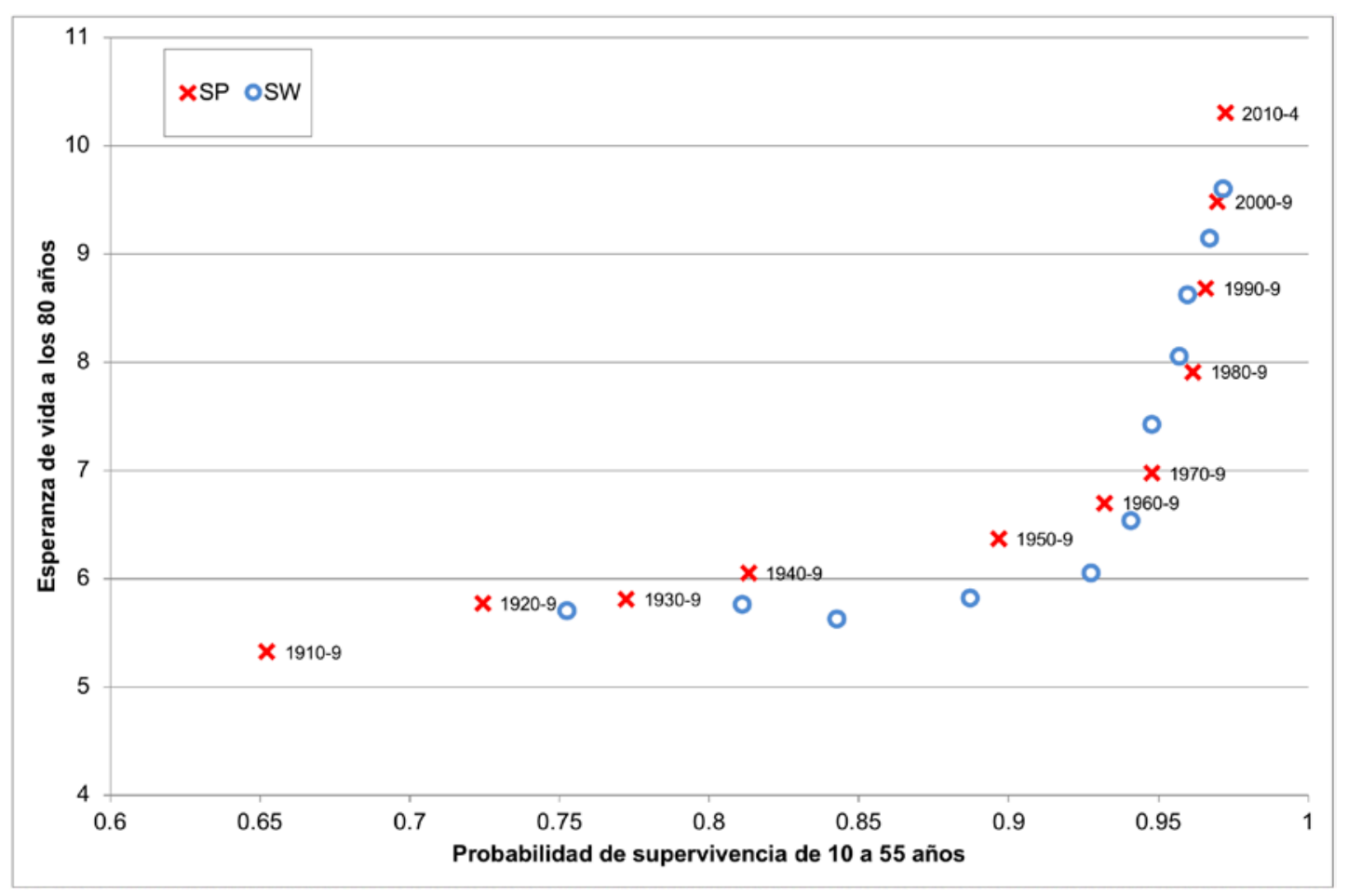

Fuente: elaboración propia a partir de datos de HMD

Figura 2. Relación entre la esperanza de vida a los 80 años y la supervivencia de los 10 a los 55 años en España y Suecia. Varones

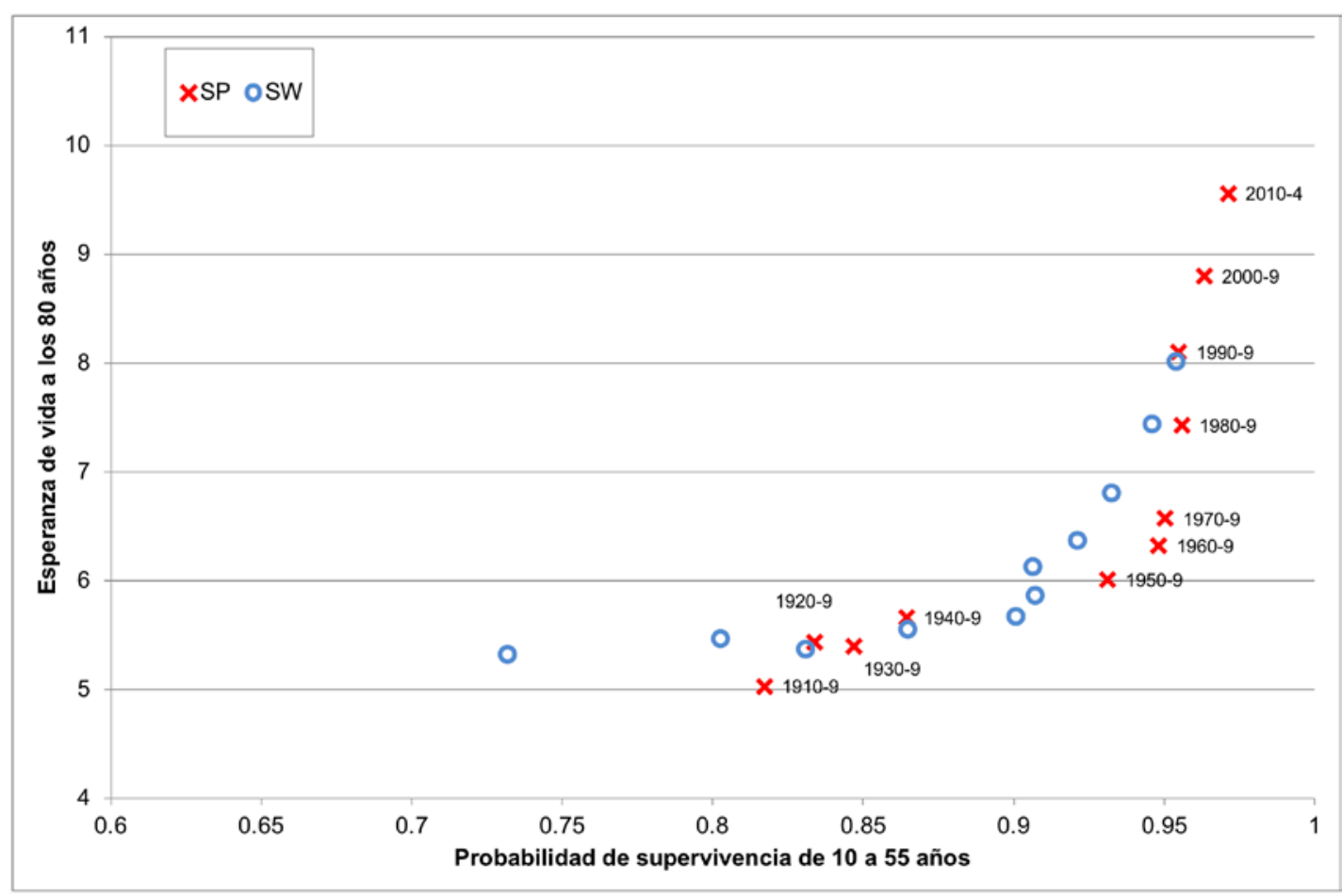

Fuente: elaboración propia a partir de datos de HMD 
Tabla 2. Ratio de defunciones a 105+ y 100+ años de edad y $110+$ y $105+$ años de edad. España y Suecia. 1910-2014. Mujeres y varones

\begin{tabular}{|c|c|c|c|c|c|c|c|c|c|c|c|c|}
\hline \multirow{3}{*}{ Año } & \multicolumn{6}{|c|}{ Ratio de defunciones $105+$ y $100+$} & \multicolumn{6}{|c|}{ Ratio de defunciones $110+$ y $105+$} \\
\hline & \multicolumn{3}{|c|}{ Mujeres } & \multicolumn{3}{|c|}{ Varones } & \multicolumn{3}{|c|}{ Mujeres } & \multicolumn{3}{|c|}{ Varones } \\
\hline & España & Suecia & Dif. & España & Suecia & Dif. & España & Suecia & Dif. & España & Suecia & Dif. \\
\hline 1910-1919 & 9,46 & 3,54 & 5,92 & 5,93 & 0,00 & 5,93 & 2,03 & 0,00 & 2,03 & 0,00 & & - \\
\hline 1920-1929 & 6,86 & 0,65 & 6,21 & 3,51 & 3,03 & 0,48 & 0,00 & 0,00 & 0,00 & 0,00 & 0,00 & 0,00 \\
\hline 1930-1939 & 11,35 & 2,45 & 8,90 & 10,89 & 2,78 & 8,11 & 9,64 & 0,00 & 9,64 & 9,96 & 0,00 & 9,96 \\
\hline 1940-1949 & 10,40 & 3,66 & 6,74 & 8,77 & 2,41 & 6,36 & 16,68 & 0,00 & 16,68 & 10,49 & 0,00 & 10,49 \\
\hline 1950-1959 & 12,47 & 1,59 & 10,88 & 12,62 & 1,71 & 10,91 & 16,08 & 0,00 & 16,08 & 17,34 & 0,00 & 17,34 \\
\hline 1960-1969 & 9,10 & 4,02 & 5,08 & 9,44 & 1,90 & 7,54 & 19,42 & 0,00 & 19,42 & 28,21 & 0,00 & 28,21 \\
\hline 1970-1979 & 10,91 & 3,54 & 7,37 & 15,49 & 3,13 & 12,36 & 27,61 & 0,00 & 27,61 & 48,43 & 0,00 & 48,43 \\
\hline 1980-1989 & 8,60 & 5,39 & 3,21 & 6,85 & 2,63 & 4,22 & 4,20 & 1,06 & 3,14 & 3,60 & 0,00 & 3,60 \\
\hline 1990-1999 & 7,74 & 5,56 & 2,18 & 6,77 & 4,31 & 2,46 & 3,65 & 2,96 & 0,69 & 4,37 & 0,00 & 4,37 \\
\hline 2000-2009 & 6,40 & 5,74 & 0,66 & 5,37 & 3,73 & 1,64 & 3,30 & 2,99 & 0,31 & 2,55 & 2,50 & 0,05 \\
\hline 2010-2014 & 6,77 & 5,42 & 1,35 & 5,08 & 2,61 & 2,47 & 2,99 & 1,69 & 1,30 & 2,97 & 0,00 & 2,97 \\
\hline
\end{tabular}

Leyenda: Dif. = Diferencia entre España y Suecia.

Para ratio de defunciones a 105+ y 100+ años de edad. (0,0-5,9), buena calidad; (6,0-9,9), calidad aceptable; $(10,0-14,9)$, calidad aceptable, pero con reservas; $(\geq 15,0)$,

Para ratio de defunciones a 110+ y 105+ años de edad. (0-9,9), buena calidad; (10,0-14,9); calidad aceptable; (15,0$24,9)$, calidad aceptable, pero con reservas; $(\geq 25,0)$,

Fuente: elaboración propia a partir de datos de HMD

El segundo indicador para medir la exageración de edades de fallecimiento es el ratio entre el número de defunciones a distintas edades: 105+ frente a 100+, y 110+ frente a 105+. Por un lado, los ratios de defunciones de 105+ años respecto a las de 100+ años en España varían notablemente, mientras que en Suecia nunca superan el nivel de 6 a lo largo de todo el período de estudio. Tanto en mujeres como en varones los datos son de baja calidad hasta la década de los setenta. Desde entonces, los datos de las mujeres han presentado una calidad aceptable hasta 2014, y los datos de los varones tenían calidad aceptable en 1980-1999 y buena calidad en 20002014 (Tabla 2).

Por otro lado, la interpretación de los ratios de las defunciones de supercentenarios (110+ años) con respecto a las de semisupercentenarios (105+ años) se complica debido a la falta de defunciones a esas edades. De hecho, no ha sido posible calcular el indicador para algunos períodos en Suecia, ya que bien el numerador, bien el denominador eran cero (Tabla 2). Así, tanto en mujeres como en varones los datos son de mala calidad hasta 1980, cuando deja de haber indicios de exageración de edades. Nótese que esa fecha incluye a las generaciones que, 
habiendo cumplido más de 105 o más de 110 años, nacieron bajo el registro obligatorio de su nacimiento. De esta manera, la fiabilidad de los datos debe aumentar.

En suma, todos estos resultados coinciden con los anteriores, lo que indica que en España ha existido un problema de exageración de edades de fallecimiento hasta que prácticamente finaliza la década de los años setenta, si bien a partir de entonces ese problema desaparece, al menos entre las defunciones a edades inferiores a 105 años.

\subsection{Redondeo o preferencia por un dígito en la edad de defunción}

El uso de pirámides de población permite realizar una aproximación exploratoria al problema del redondeo de edades en la población española. En la Figura 3 podemos ver que en 1910 y 1940 el número de personas con 30, 40, 50, 60 y 70 años es notablemente mayor que el de las edades contiguas. Ya en 1970 sólo se observa un ligero saliente para las mujeres (los de 30 años no deben considerarse, ya que se corresponde con el baby boom de 1940, primer año de post guerra). Al trasladar este análisis previo a la estructura por edad de las defunciones (Figura 4), en 1910-1919 y 1940-1949 se repite el patrón de redondeo a los 50, 60, 70, 80 y 90 años de edad de defunción; en cambio, en 1970-1979 ya no aparecen esas sobreestimaciones en las edades acabadas en cero.

Para confirmar estos indicios de redondeo, el primer indicador que utilizamos es el índice de Whipple, que calculamos para nonagenarios y para centenarios. En el caso de los nonagenarios, se aprecian ciertos problemas de calidad de 1910 a 1959 en el caso de las mujeres, y de 1910 a 1949 en el caso de los varones, situación que se confirma con las diferencias relativas respecto al golden standard, que alcanzan hasta un $30 \%$ (Tabla 3). Desde 1960 la calidad de los datos españoles se equipara a la de los suecos. 
Figura 3. Estructura por edad de la población de España. 1910, 1940, 1970, 2014
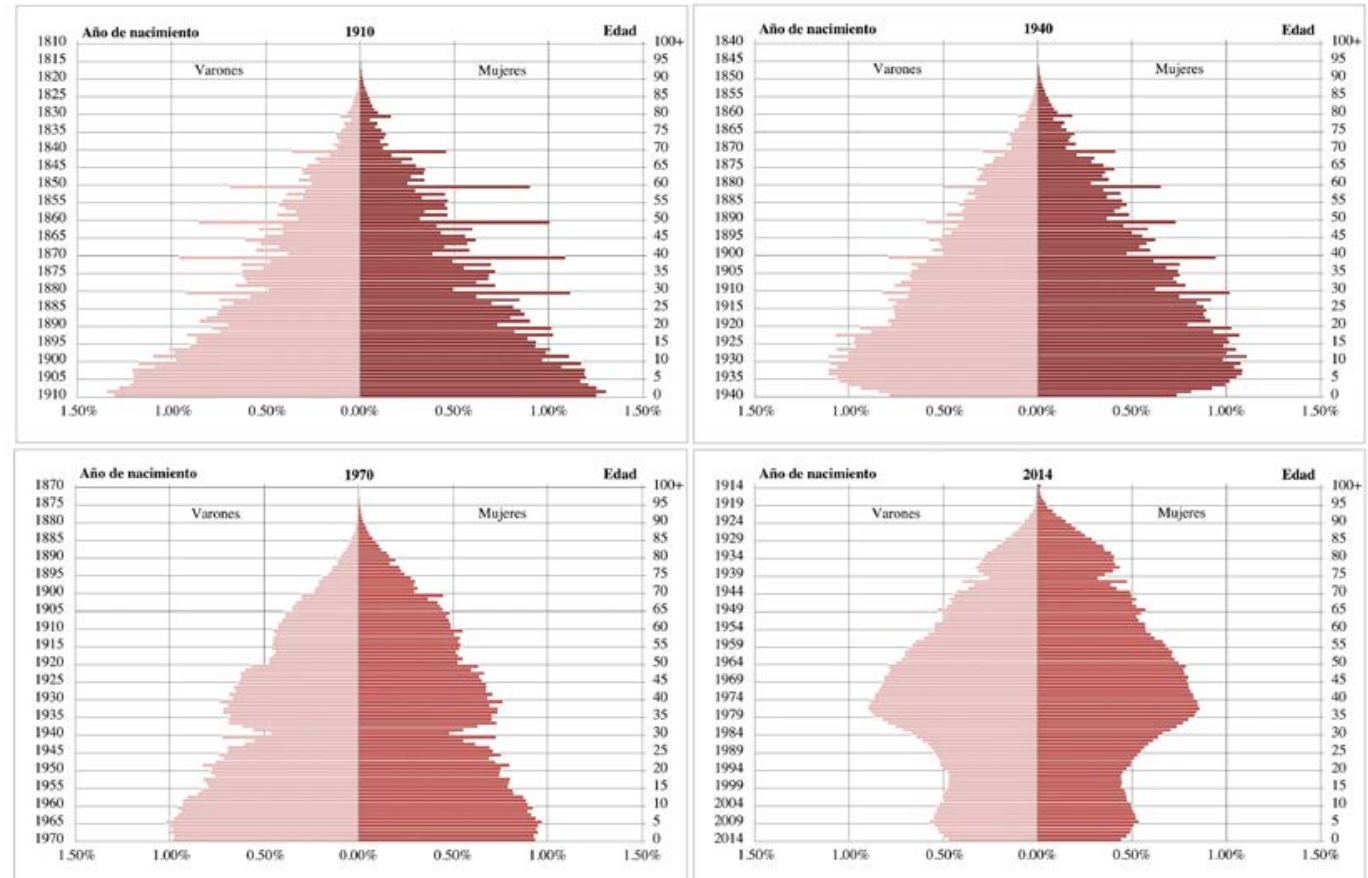

Nota: en este caso no se toman las poblaciones por décadas porque las poblaciones expuestas al riesgo por décadas -las únicas disponibles- están suavizadas y corregidas, de modo que no se vislumbra el redondeo.

Fuente: elaboración propia a partir de datos de HMD

Figura 4. Estructura por edad de las defunciones en España. 1910-1919, 1940-1949, 1970-1979, 2010-2014

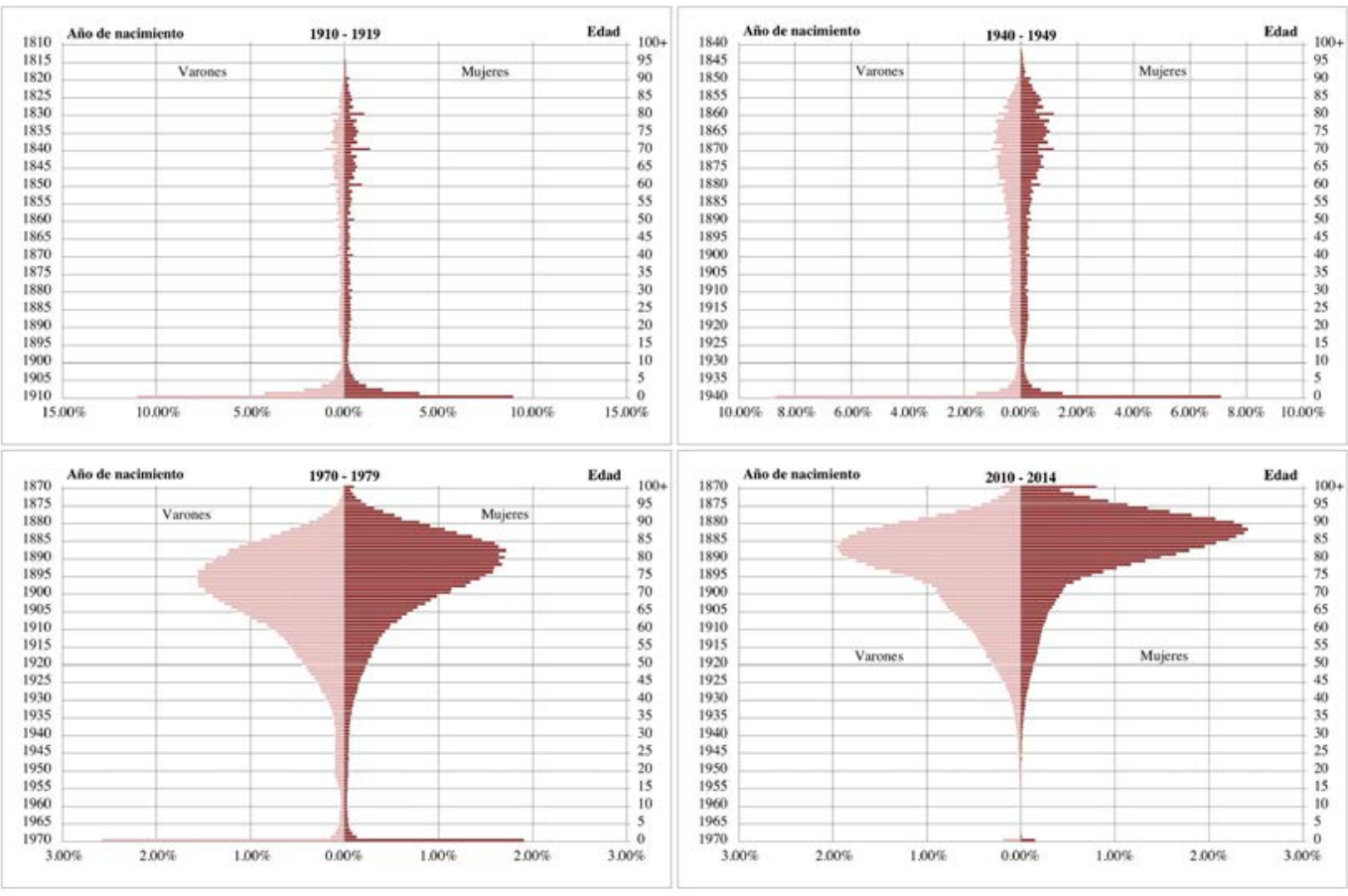

Fuente: elaboración propia a partir de datos de HMD 


\section{Tabla 3. Índice de whipple para nonagenarios y centenarios.}

\section{España vs. Suecia. 1910-2014. Mujeres y varones}

\begin{tabular}{|c|c|c|c|c|c|c|c|c|c|c|c|c|}
\hline \multirow{3}{*}{ Año } & \multicolumn{5}{|c|}{ Nonagenarios } & \multicolumn{5}{c|}{ Centenarios } \\
\cline { 2 - 15 } & \multicolumn{3}{|c|}{ Mujeres } & \multicolumn{3}{|c|}{ Varones } & \multicolumn{3}{c|}{ Mujeres } & \multicolumn{3}{c|}{ Varones } \\
\cline { 2 - 15 } & España & Suecia & Dif. & España & Suecia & Dif. & España & Suecia & Dif. & España & Suecia & Dif. \\
\hline $1910-1919$ & 131,73 & 99,45 & 32,46 & 116,52 & 100,63 & 15,80 & 107,88 & 92,45 & 16,69 & 97,03 & 91,78 & 5,71 \\
\hline $1920-1929$ & 129,50 & 101,49 & 27,60 & 113,56 & 98,72 & 15,04 & 111,22 & 92,58 & 20,12 & 97,40 & 83,76 & 16,28 \\
\hline $1930-1939$ & 122,89 & 98,12 & 25,24 & 110,39 & 100,27 & 10,09 & 106,51 & 88,84 & 19,90 & 93,45 & 94,33 & $-0,94$ \\
\hline $1940-1949$ & 115,05 & 100,86 & 14,08 & 104,49 & 98,06 & 6,55 & 100,67 & 88,32 & 13,97 & 92,29 & 84,70 & 8,95 \\
\hline $1950-1959$ & 104,97 & 97,42 & 7,75 & 99,02 & 99,22 & $-0,21$ & 92,40 & 89,09 & 3,72 & 84,65 & 89,58 & $-5,51$ \\
\hline $1960-1969$ & 100,60 & 99,70 & 0,91 & 97,76 & 98,02 & $-0,26$ & 87,40 & 93,91 & $-6,94$ & 82,89 & 85,90 & $-3,50$ \\
\hline $1970-1979$ & 100,11 & 101,25 & $-1,13$ & 97,36 & 99,82 & $-2,46$ & 91,26 & 91,39 & $-0,14$ & 89,02 & 94,13 & $-5,43$ \\
\hline $1980-1989$ & 100,48 & 101,50 & $-1,01$ & 99,39 & 99,07 & 0,33 & 94,90 & 94,01 & 0,95 & 95,49 & 90,86 & 5,09 \\
\hline $1990-1999$ & 101,12 & 100,58 & 0,54 & 100,11 & 100,10 & 0,01 & 94,21 & 95,92 & $-1,78$ & 93,71 & 91,58 & 2,33 \\
\hline $2000-2009$ & 100,99 & 100,28 & 0,71 & 99,87 & 100,12 & $-0,25$ & 96,71 & 97,08 & $-0,38$ & 94,91 & 92,69 & 2,39 \\
\hline $2010-2014$ & 101,66 & 100,87 & 0,78 & 100,80 & 101,21 & $-0,41$ & 97,03 & 95,49 & 1,62 & 94,10 & 92,43 & 1,81 \\
\hline
\end{tabular}

Leyenda: Dif. = Diferencia relativa entre España y Suecia.

Para el índice de Whipple. ( $\leq$ 105), datos muy fiables; (105-110), datos fiables pero con reservas; (111-125), datos aceptables; (126-175),

datos poco fiables; $(\geq 176)$, datos no fiables.

Para la diferencia relativa. (0,0-4,9), mujeres y varones, buena calidad; (5,0-8.9) mujeres, (5,0-9.9), varones, calidad aceptable; $(9,0-11,9)$, mujeres, $(10,0-14,9)$, varones, calidad relativamente aceptable; $(\geq 12,0)$, mujeres, $(\geq 15,0)$, varones,

Fuente: elaboración propia a partir de datos de HMD

Los centenarios mantienen una tendencia similar. El índice de Whipple muestra que las mujeres presentan datos de baja calidad hasta la década de los años treinta, mientras que la comparativa con el golden standard revela problemas de redondeo también en 1940-1949 y 1960-1969. Los varones tienen una evolución irregular. Si bien el índice de Whipple nos dice que no hay redondeo de edades, al comparar con Suecia vemos que hay huellas leves en 1910-1919, 1940-1959 e incluso en 1970-1989, y profundas en 1920-1929 (Tabla 3). Debemos ser prudentes en la interpretación de los datos de varones y en la comparación con las mujeres, ya que las bajas cifras de defunciones de centenarios podrían tergiversar el índice tanto en España como en Suecia.

El índice de redondeo de edades es el segundo indicador que usamos para medir la preferencia por un dígito en la edad de defunción (Tabla 4). Tanto los datos de mujeres como los de varones tienen serios problemas de calidad en las edades de defunción acabadas en cero (70, 80 y 90) hasta la década de 1950-59. Las centenarias mujeres, en cambio, presentan redondeo sólo hasta 1940-1949, mientras que los centenarios varones sólo lo hacen en 1920-29 y 1940-49, lo que 
coincide en parte con el índice de Whipple tanto en el resultado como en la precaución en la interpretación. En el caso de las edades acabadas en 5 (75, 85 y 95), hay redondeo hasta los años cuarenta en el caso de las mujeres, y hasta los años treinta en el caso de los varones (excepto en la edad 95).

\section{Tabla 4. Índice de redondeo de edades de defunción}

para 70, 75, 80, 85, 90, 95 y 100 años de edad. España. 1910-2014. Mujeres y varones

\begin{tabular}{|c|c|c|c|c|c|c|c|c|c|c|c|c|c|c|}
\hline \multirow{2}{*}{ Año } & \multicolumn{7}{|c|}{ Mujeres } & \multicolumn{7}{|c|}{ Varones } \\
\hline & 70 & 75 & 80 & 85 & 90 & 95 & 100 & 70 & 75 & 80 & 85 & 90 & 95 & 100 \\
\hline 1910-1919 & 2,27 & 1,28 & 2,23 & 1,20 & 1,97 & 1,20 & 0,99 & 1,79 & 1,19 & 1,76 & 1,12 & 1,65 & 1,09 & 0,86 \\
\hline 1920-1929 & |1,99 & 1,27 & 1,99 & 1,21 & 1,87 & 1,19 & 1,29 & 1,60 & 1,19 & 1,62 & 1,13 & 1,51 & 1,05 & 1,20 \\
\hline 1930-1939 & $\mid 1,72$ & 1,22 & 1,72 & 1,16 & 1,65 & 1,12 & 1,31 & 1,44 & 1,16 & 1,41 & 1,11 & 1,38 & 1,04 & 1,09 \\
\hline 1940-1949 & 1,46 & 1,15 & 1,47 & 1,11 & 1,43 & 1,08 & 1,17 & 1,26 & 1,10 & 1,24 & 1,07 & 1,21 & 1,01 & 1,17 \\
\hline 1950-1959 & 1,20 & 1,04 & 1,21 & 1,03 & 1,22 & 1,01 & 1,03 & 1,11 & 1,03 & 1,11 & 1,01 & 1,12 & 0,96 & 0,97 \\
\hline 1960-1969 & 1,04 & 1,02 & 1,08 & 1,02 & 1,08 & 0,96 & 1,01 & 1,02 & 1,01 & 1,04 & 1,01 & 1,06 & 0,95 & 0,94 \\
\hline 1970-1979 & 1,04 & 1,01 & 1,02 & 1,01 & 1,05 & 1,00 & 1,09 & 1,02 & 1,01 & 1,01 & 1,00 & 1,02 & 1,01 & 1,18 \\
\hline 1980-1989 & 1,00 & 1,00 & 1,03 & 1,02 & 1,03 & 1,03 & 1,13 & 1,01 & 1,01 & 1,03 & 1,02 & 1,03 & 1,04 & 1,31 \\
\hline 1990-1999 & 1,00 & 1,00 & 1,02 & 1,02 & 1,04 & 1,03 & 1,04 & 1,01 & 1,00 & 1,00 & 1,02 & 1,03 & 1,05 & 1,09 \\
\hline 2000-2009 & 1,00 & 1,01 & 1,02 & 1,01 & 1,02 & 1,04 & 1,05 & 1,00 & 1,01 & 1,02 & 1,01 & 1,02 & 1,04 & 1,09 \\
\hline 2010-2014 & 1,02 & 1,00 & 1,01 & 1,02 & 1,04 & 1,02 & 1,07 & 1,02 & 0,99 & 1,02 & 1,02 & 1,04 & 1,02 & 1,11 \\
\hline
\end{tabular}

Leyenda: $(<1,1)$, calidad buena o aceptable, no hay redondeo; $(1,1-1,2)$, niveles moderados de redondeo; $(>1,2)$,

Fuente: elaboración propia a partir de datos de HMD

\section{Discusión}

Las evidencias disponibles sugieren ocho explicaciones a la deficiente calidad de las estadísticas de defunciones del Registro civil, que conviene discutir a la luz de los resultados expuestos.

En primer lugar, a pesar del gran cambio que experimentó España, el Registro civil que deja la legislación de 1870 se mantiene con muy pocos cambios hasta la Ley de 8 de junio de 1957, del Registro Civil (y posterior Reglamento de 14 de noviembre de 1958). El INE propuso un nuevo modelo de boletín estadístico de defunción más completo que los anteriores para así poder atender la creciente demanda de datos sociosanitarios. Su complejidad provocó un doble efecto: por un lado, aumentó la falta de respuesta; por otro, mejoró la calidad de la información recogida.

En segundo lugar, la ley de 1870 exigía que en el acta de nacimiento se determinara la filiación legítima o ilegítima del inscrito, si bien no existía equiparación legal en las constituciones de 1869 y 1876. Esto no cambió hasta la constitución de 1931 (artículo 43), que prohibió expresar esa 
circunstancia vital en el registro. El estigma y la baja consideración social de los hijos considerados ilegítimos, que se estimaron en casi un 5 \% en la primera década del siglo XX (Livi-Bacci, 1968), también pudieron incidir en el subregistro de nacimientos.

En tercer lugar, la ley de 1870 impuso la obligatoriedad de presentar al recién nacido en el registro como garantía de la veracidad del nacimiento, exigencia que se eliminó con el artículo 328 del Código Civil de 1889, para así evitar peligros para la salud de los bebés. Esta imposición puede relacionarse con el subregistro de nacimientos y, en el caso de los hijos considerados ilegítimos, como una dificultad añadida.

En cuarto lugar, en muchos municipios hubo un subregistro de eventos. Un primer caso es la omisión de nacimientos: el Instituto Geográfico y Estadístico estimaba que en 1905 alcanzó el 6 \%. Livi-Bacci (1968) cifró este subregistro en un 4 \% entre 1891 y 1900, mientras que Leasure (1962) lo hizo en un $11 \%$ en 1900 y en un $6 \%$ en 1920. Un segundo caso, relacionado con el anterior, ilustra la deficiente cobertura femenina del Registro civil: en los períodos 1861-1863, 1900-1910 y 1911-1920 el ratio de masculinidad al nacimiento en España se estimaba en 110 (Livi-Bacci, 1968), que superaba notablemente al ratio biológico de 105-107 varones por cada 100 mujeres. En algunas regiones como Murcia o Asturias el subregistro femenino fue mucho más acusado, con ratios de masculinidad de 123 y 118 en 1900-1903. La ausencia de explicación por razones biológicas sólo deja viables factores humanos que apuntan a una falta de cobertura del Registro civil, especialmente en los municipios más pequeños (Viciana, 1988). Asimismo, la comparativa con Suecia -nuestro golden standard-y otros países europeos muestra claras incoherencias. Esta falta de cobertura se traslada también a las defunciones de la primera mitad del siglo XX, ya que no se tiene demasiada información sobre el funcionamiento de los circuitos de su declaración estadística, indicador evidente de subregistro (Viciana, 1988).

En quinto lugar, la exageración de edades a la defunción en España hasta los años setenta se explica por tres factores. Primero, por las bajas tasas de alfabetización que hubo hasta más de mediados de siglo XX: entre los mayores de diez años, en 1887 la tasa era del $35 \%$, en 1910 del $48 \%$, y del $77 \%$ en 1940. En las edades de 21 a 50 -que podemos considerar "fértiles" para ambos sexos- las tasas se situaban entre el $30 \%$ y el $40 \%$ en 1887 ; entre el $44 \%$ y el $55 \%$ en 1910; y entre el $72 \%$ y el $87 \%$ en 1940 (de Gabriel, 1997). Cabe destacar que los progresos se producen sobre todo entre 1910 y 1940, principalmente entre los niños, lo que redunda en una posterior mejora de las inscripciones de eventos vitales de esas generaciones y, por lo tanto, de la calidad de los datos de mortalidad. Segundo, el ya consabido imperativo de que sea un tercero quien inscriba la defunción, con el consiguiente aumento de probabilidad de que se cometan errores -más si cabe con las altas tasas de analfabetismo previamente reseñadas. Y tercero, sobre 
todo para el caso de los censos de población, Ewbank (1981) alude a la progresiva pérdida de memoria de los ancianos, que tenderían así a la sobreestimación de sus edades. Por su parte, Pastor (1999) afirma que en el período 1900-1930 se produjeron subrecuentos de ancianos tanto en los censos como en las estadísticas de defunciones, lo que puede justificarse por declaraciones erróneas de las edades -cronológica y de fallecimiento- que se tradujeron en una sistemática exageración de edades a partir de los 60 años de edad.

En sexto lugar, las afirmaciones sobre las edades de los centenarios y supercentenarios en España tienen que tomarse con mucha cautela por tres razones. Primero, la escasez de datos fiables hasta finales del siglo XX, especialmente entre los varones. Segundo, la tasa de verificación de defunciones de personas que registraron su muerte con 110 años o más fue de alrededor de un 25\% en el período 1987-2009 (Gómez Redondo y García González, 2010, 2011). En este sentido se complica sobremanera la comprobación de la fecha de nacimiento para aquellas personas que nacieron previamente a la implantación del Registro civil. Tercero, si se pretendiera comparar con el golden standard, no hay defunciones de supercentenarias hasta 1980 y solo hay una de un supercentenario varón hasta 2003 (International Database on Longevity, 2016), lo que imposibilita la comparación.

En séptimo lugar, los indicadores de redondeo muestran que en España ha habido problemas de fiabilidad en las edades de defunción acabadas en 0 y 5 hasta la década de los años sesenta. Hay diversas razones que lo explican. Primero, la introducción del nuevo boletín de defunción en 1957, que preguntaba por la fecha de nacimiento en lugar de por la edad de defunción, limitando así las potenciales inexactitudes en su declaración. Segundo, Reher y Valero (2005) apuntan que el Registro civil no se consolidó plenamente hasta el primer cuarto del siglo XX: no tenía las suficientes herramientas ni recursos para garantizar una correcta inscripción de los nacimientos y de las defunciones, las fluctuaciones de las administraciones estatal y local generaban problemas de coordinación, y el índice de alfabetización de la población era muy bajo. Tercero, la edad modal de muerte durante la primera parte del siglo XX, una vez suavizado el efecto redondeo, se estimaba en 75 años entre las mujeres y a los 70 años en los varones (Génova, 2007), lo que podría proporcionar un estímulo latente a los inscriptores. Cuarto, y en relación con lo anterior, los fallecidos con más de 70 años de edad de 1910 a 1949 nacieron desde antes de 1879, por lo que se corresponden en su mayor parte con generaciones nacidas previamente a la implantación del Registro civil en 1870 y que no inscribieron su nacimiento. En estos casos no era posible, por lo tanto, disponer de la fecha de nacimiento real para comprobar la verdadera edad de defunción sin tener que acudir a un registro parroquial. Quinto, al igual que ocurre con la exageración de edades, tuvieron importancia el bajo nivel educativo de la población, la necesidad de que un 
tercero inscribiera la defunción y la pérdida de memoria de los ancianos (en el caso de los datos censales) (Ewbank, 1981).

En octavo lugar, la declaración de la edad de defunción experimentó una evolución paradójica: hasta la década de los años cincuenta el porcentaje de la categoría "no consta edad" se situó por debajo del 1\%; en cambio, en el período 1950-1974 alcanzó valores entre el 30 \% y el 56 \% (Viciana, 1988). Una posible explicación la encontramos en el boletín de defunción. El cuestionario preguntaba directamente por la edad de defunción hasta 1957, año en el que esa variable se cambió por fecha de nacimiento. La declaración específica de la edad está muy sujeta a la imprecisión, ya que su aparente facilidad puede provocar fallos u olvidos por parte de los que rellenan el boletín; en cambio, la fecha de nacimiento es una variable considerablemente más compleja, lo que explicaría el aumento de la proporción de "no consta edad". El efecto inmediato es una mejora de la calidad de las estadísticas de defunción, ya que se evitan los problemas aparejados a la vaguedad de la declaración de la edad de muerte -caso del redondeo y la exageración- y de la evidente limitación de toda declaración de la edad de fallecimiento: el sujeto directamente implicado nunca la puede confirmar por sí mismo y lo delega en un representante.

\section{Conclusiones}

El presente trabajo ha mostrado cómo entre 1910 y 2014 se ha producido una considerable mejora de la calidad de las estadísticas españolas de mortalidad a las edades avanzadas. Destacan sobre todo los progresos que se produjeron a partir de mediados de siglo XX, que provocan que a partir de la década de los ochenta prácticamente desaparezcan los principales problemas que aquejaban a esos datos: la exageración y el redondeo de edades a la defunción, equiparándose su calidad a la del golden standard Suecia.

Este progreso se explica básicamente por tres factores que se retroalimentan. Primero, los avances se relacionan directamente con la modernización de España en los ámbitos social, políticoinstitucional, demográfico, económico y geográfico. Destacan especialmente el incremento de la alfabetización de la población española, que redunda en inscripciones vitales más fiables, y el perfeccionamiento de la descentralización de funciones y la cesión de competencias a las entidades locales. Segundo, en clara vinculación con lo anterior, sobre todo a partir de la ley de 1957, el Registro civil también se va modernizando paulatinamente: se produce una especialización de funcionarios, una mayor cobertura geográfica, y una adecuación de la norma y los boletines a la realidad del país. Y tercero, mejora la recolección y el tratamiento de las estadísticas vitales por parte de los profesionales médicos, de los registros civiles y del Movimiento Natural de la Población elaborado por el INE. 
Ahora bien, a pesar del considerable esfuerzo y consecuente progreso, las estadísticas de mortalidad a edades avanzadas presentan aún un importante punto débil, que se encuentra en las edades más avanzadas de defunción: centenarios y supercentenarios. Los actuales procesos de verificación de las edades de personas fallecidas con 110 años o más han mostrado relativos índices de éxito y algunas dificultades insalvables relacionadas con los procesos migratorios internos, de modo que todavía hay que mantener ciertas reservas tanto en el recuento de estas poblaciones como en sus verdaderas edades de fallecimiento.

Por último, los resultados de este estudio dejan abiertas tres futuras líneas de investigación. Primero, su consideración para la mejora la calidad de las estadísticas de mortalidad y la suavización de los datos históricos. Segundo, se puede extender el estudio de la calidad de las estadísticas de mortalidad a otras edades, a otros períodos de tiempo o a una profundización en las diferencias por sexo. Y tercero, la estandarización del proceso permite comparativas internacionales y, dadas las características territoriales de España, comparativas entre regiones o entidades locales. 


\section{Bibliografía}

Anderson, B. A., y Silver, B. D. (1997). Issues of data quality in assessing mortality trends and levels in the New Independent States. En J. L. Bobadilla, C. A. Costello y F. Mitchell (Eds.), Premature death in the new independent states (pp. 120-154). Washington: National Academy Press.

Recuperado en mayo de 2017, de http://www.ncbi.nlm.nih.gov/books/NBK233395/pdf/Bookshelf_NBK233395.pdf

Arbelo Curbelo, A. (1962). La mortalidad de la infancia en España: 1901-1952. Madrid: Dirección General de Sanidad.

Bennett, N. G., y Garson, L. K. (1983). The centenarian question and old-age mortality in the Soviet Union, 1959-1970. Demography, 20(4), 587-606.

Bernabeu Mestre, J. (1992). Fuentes para el estudio de la mortalidad en la España del siglo XIX. Las estadísticas demográfico-sanitarias. En Ministerio de Sanidad y Consumo (Ed.), Las estadísticas demográfico-sanitarias. I Encuentro Marcelino Pascua (pp. 27-46). Madrid: Ministerio de Sanidad y Consumo.

Blanes, A. (2007). La mortalidad en la España del siglo XX. Análisis demográfico y territorial (Tesis doctoral). Recuperado en mayo de 2017, de http://sociales.cchs.csic.es/jperez/PDFs/Blanes2007.pdf

Bourbeau, R., y Desjardins, B. (2002). Dealing with problems in data quality for the measurement of mortality at advanced ages in Canada. North American Actuarial Journal, 6(3), 1-13.

Bourbeau, R., y Desjardins, B. (2006). Mortality at Extreme Ages and Data Quality: The Canadian Experience. En J. M. Robine et al. (Eds.), Human Longevity, Individual Life Duration and the Growth of the Oldest Old Population (pp. 167-185). Berlín: Springer.

Bourbeau, R., y Lebel, A. (2000). Mortality Statistics for the Oldest-Old: An Evaluation of Canadian Data. Demographic Research, 2(2). Recuperado en mayo de 2017, de http://www.demographicresearch.org/volumes/vol2/2/2-2.pdf

Brel Cachón, M. P. (1999). Comparación de los libros parroquiales y de los registros civiles. Una aportación a la validez de las fuentes demográficas a finales del siglo XIX. Boletín de la Asociación de Demografía Histórica, XVII(2), 91-114. Recuperado en mayo de 2017, de http://www.adeh.org/?q=es/system/files/Revista/1999\%2C\%202/MP\%20Brel\%2C\%20XVII\%2 C\%20II\%2C\%201999\%2C\%20pp\%2091-114.pdf

Cheung, S. L. K., y Robine, J. M. (2007). Increase in common longevity and the compression of mortality: the case of Japan. Population Studies, 61, 85-97. 
Coale, A. J., y Kisker, E. E. (1986). Mortality crossovers: reality or bad data? Population Studies, $40,389-401$.

Cusidó i Vallverdú, T. A. (2012). El Moviment natural de la població. Història, conceptes i anàlisi crítica dels continguts (Tesis doctoral). Recuperado en mayo de 2017, de http://www.tesisenred.net/handle/10803/96811

Ewbank, D. C. (1981). Age misreporting and age-selective underenumeration: sources, patterns, and consequences for demographic analysis» en Committee on Population and Demography Report n. ${ }^{\circ}$ 4. Washington D.C.: National Academy Press.

Gabriel, N. de (1997). Alfabetización, semialfabetización y analfabetismo en España (1860-1991). Revista Complutense de Educación, 8(1), 199-231.

García González, J. M. (2014). ¿Por qué vivimos más? Descomposición por causa de la esperanza de vida española de 1980 a 2009. Revista Española de Investigaciones Sociológicas, 148, 39-60. Recuperado en mayo de 2017, de http://reis.cis.es/REIS/PDF/REIS_148_031413365444307.pdf

Génova, R. (2007). Longevidad y compresión de la mortalidad en la población española. Comunicación presentada en el IX Congreso Español de Sociología. Barcelona, 13-15 de septiembre.

Gómez Redondo, R. (1984). Mortalidad infantil real frente a mortalidad infantil legal. Boletín de la Asociación de Demografía Histórica, I/(1), 6-19. Recuperado en mayo de 2017, de http://www.adeh.org/?q=es/system/files/Revista/1984-

1/R\%20G\%C3\%B3mez\%2C\%20II\%2C\%20i\%2C\%201984\%2C\%20pp\%206-19.pdf

Gómez Redondo, R. (1992). La mortalidad infantil en España en el siglo XX. Madrid: Centro de Investigaciones Sociológicas.

Gómez Redondo, R., y García González, J. M. (2010). Emergence and verification of supercentenarians in Spain. En H. Maier, J. Gampe, B. Jeune, J. M. Robine y J. W. Vaupel (Eds.), Supercentenarians (pp. 151-171). Heidelberg: Springer. Recuperado en mayo de 2017, de http://www.demogr.mpg.de/books/drm/007/2-7.pdf

Gómez Redondo, R., y García González, J. M. (2011). From Montreal to Madrid: updating the data of Spanish supercentenarians. Comunicación presentada en el 8th Workshop on Supercentenarians. Madrid, 27-28 de enero.

Human Mortality Database (2016). Data for Spain and Sweden, 1910-2014 [Base de datos]. Recuperado en mayo de 2017, de http://www.mortality.org 
International Database on Longevity (2016). Data for Sweden [Base de datos]. Recuperado en mayo de 2017, de http://www.supercentenarians.org

Instituto Geográfico y Estadístico (1906). Movimiento natural de la población de España. Madrid: Instituto Geográfico y Estadístico.

Instituto Nacional de Estadística (2016). Indicadores demográficos básicos. Recuperado en mayo de 2017, de http://www.ine.es

Jdanov, D. A., y Jasilionis, D. (2010). Mortality at old ages: evaluation of data quality. Comunicación presentada en la European Population Conference 2010. Viena, 1-4 de septiembre.

Jdanov, D. A., Jasilionis, D., Soroko, E. L., Rau, R., y Vaupel, J. W. (2008). Beyond the KannistoThatcher Database on Old Age Mortality: An Assesment of Data Quality at Advanced Ages (Working Paper). Max Planck Institute for Demographic Research, n WP-2008-13. Recuperado en mayo de 2017, de http://www.demogr.mpg.de/papers/working/wp-2008-013.pdf

Kannisto, V. (1994). Development of oldest-old Mortality, 1950-1990. Evidence from 28 developed countries. En B. Jeune y J. W. Vaupel (Eds.), Odense Monographs on Population Aging 1.

Odense: Odense University Press. Recuperado en mayo de 2017, de http://www.demogr.mpg.de/Papers/Books/Monograph1/start.htm

Kannisto, V. (1999). Assessing the Information on Age at Death of Old Persons in National Vital Statistics» en Validation of exceptional longevity. En B. Jeune y J. W. Vaupel (Eds.), Odense Monographs on Population Aging 6 (pp. 240-249). Odense: Odense University Press. Recuperado en mayo de 2017, de http://www.demogr.mpg.de/books/odense/6/15.htm

Kannisto-Thatcher Database on Old-Age Mortality (2016). Data for Spain and Sweden [Base de datos]. Recuperado en mayo de 2017, de http://www.demogr.mpg.de/databases/ktdb Leasure, J. W. (1963). Factors involved in the decline of fertility in Spain 1900-1950. Population Studies, 16(3), 271-285.

Livi-Bacci, M. (1968). Fertility and nuptiality changes in Spain from the late 18th to the early 20th century. Parts 1\&2. Population Studies, 22(1), 83-102 \& 211-234.

Lundström, H. (1995). Record Longevity in Swedish Cohorts Born since 1770. En B. Jeune y J. W. Vaupel (Eds.), Exceptional longevity: from prehistory to present. Odense Monographs on Population Aging 2 (pp. 67-74). Odense: Odense University Press. Recuperado en mayo de 2017, de http://www.demogr.mpg.de/Papers/Books/Monograph2/start.htm

Nicolau, R. (2005). Población, salud y actividad. En A. Carreras y X. Tafunell (Coords.), Estadísticas históricas de España. Siglos XIX-XX (pp. 77-154). Bilbao: Fundación BBVA. 
Recuperado en mayo de 2017, de

http://www.fbbva.es/TLFU/dat/DE_2006_estadisticas_historicas.pdf

Pascua, M. (1934a). La mortalidad infantil en España. Madrid: Dirección General de Sanidad.

Pascua, M. (1934b). Mortalidad específica en España. Madrid: Dirección General de Sanidad.

Pastor García-Quismondo, F. (1999). Declaración errónea de la edad de adultos y ancianos en España: evaluación de la consistencia de los datos para el análisis de la mortalidad, 1900-1930. Revista de Demografía Histórica, XVII(2), 29-60. Recuperado en mayo de 2017, de hHp://www.adeh.org/?q=es/system/files/Revista/1999\%2C\%202/F\%20Pastor\%2C\%20XVII\% 2C\%20II\%2C\%201999\%2C\%20pp\%2029-60.pdf

Pérez Moreda, V. (1980). La crisis de mortalidad en la España interior: siglos XVI-XIX. Madrid: Siglo XXI.

Preston, S.H., Elo, I. T., y Stewart, Q. (1999). Effects of age misreporting on mortality estimates at older ages. Population Studies, 53(2), 165-177.

Reher, D. S., y Valero, A. P. (2005). Fuentes de información demográfica en España. Madrid: Centro de Investigaciones Sociológicas.

Skytthe, A., y Jeune, B. (1995). Danish centenarians after 1800. En B. Jeune y J. W. Vaupel (Eds.), Exceptional longevity: from prehistory to present. Odense Monographs on Population Aging 2 (pp. 55-66). Odense: Odense University Press. Recuperado en mayo de 2017, de http://www.demogr.mpg.de/Papers/Books/Monograph2/start.htm

Skytthe, A., Hauge, G., y Jeune B., (1999). Age Validation of Reported Centenarians before 1900 in Denmark. En B. Jeune y J. W. Vaupel (Eds.), Validation of exceptional longevity. Odense Monographs on Population Aging 6 (pp. 79-96). Odense: Odense University Press. Recuperado en mayo de 2017, de http://www.demogr.mpg.de/books/odense/6/05.htm

Spoorenberg, T. (2007). La qualité des déclarations par âge: extension et application de l'indice de Whipple modifié. Population, 62, 847-859.

Thatcher, A. R., Kannisto, V., y Vaupel, J. W. (1998). The force of mortality at ages 80 to 120. En B. Jeune y J. W. Vaupel (Eds.), Odense Monographs on Population Aging 5. Odense: Odense University Press. Recuperado en mayo de 2017, de http://www.demogr.mpg.de/Papers/books/monograph5/start.htm

Thatcher, R., Kannisto, V., y Andreev, K. (2002). The survivor ratio method for estimating number at high ages». Demographic Research, 6(1), 1-18. Recuperado de http://www.demographicresearch.org/volumes/vol6/1/6-1.pdf 
Viciana, F. (1998). La transición demográfica y sanitaria en Andalucía durante el siglo XX (Tesis doctoral). Recuperado en mayo de 2017, de http://fondosdigitales.us.es/tesis/tesis/2748/latransicion-demografica-y-sanitaria-en-andalucia-durante-el-siglo-xx/

Wang, Z., Zeng, Y., Jeune, B., y Vaupel, J. W. (1999). Age Validation of Han Chinese Centenarians. En B. Jeune y J. W. Vaupel (Eds.), Validation of exceptional longevity. Odense Monographs on Population Aging 6 (pp. 195-214). Odense: Odense University Press. Recuperado en mayo de 2017, de http://www.demogr.mpg.de/books/odense/6/12.htm

Wilmoth, J. R., Andreev, K., Jdanov, D., y Glei, D. A. (2007). Methods protocol for the Human Mortality Database. Version 5 (Working Paper). Recuperado en mayo de 2017, de http://www.mortality.org/Public/Docs/MethodsProtocol.pdf 